\title{
Los periódicos gallegos en las redes sociales virtuales: presencia y posicionamiento en el nuevo escenario comunicativo
}

\author{
Xosé LóPez GARCíA \\ Universidad de Santiago de Compostela \\ xose.lopez.garcia@usc.es \\ Sara Alonso RodRíGuez \\ Universidad de Santiago de Compostela \\ sara.alonso.rodriguez@gmail.com
}

Recibido: 05/11/2012

Aceptado: 17/06/2013

\begin{abstract}
Resumen
Los periódicos gallegos priorizan el empleo de las redes sociales como canal de distribución mientras exploran otros aprovechamientos en el nuevo escenario comunicativo. El presente estudio describe y clasifica la actividad y contenidos de los diarios generalistas de mayor difusión en Galicia, junto con el nativo digital más veterano, en tres de las redes sociales con mayor penetración en la Comunidad ( $\mathrm{F} a$ cebook, Twitter y Youtube) según su presencia-promoción, la gestión de la conversación y la creación de contenidos de interés general. Los resultados alcanzados dibujan un paisaje mediático aún dominado por el tradicional modelo pasivo y unidireccional en el que las potencialidades de las redes sociales se limitan al escaparate de promoción y la distribución de contenido.
\end{abstract}

Palabras clave: redes sociales, periodismo digital, interactividad, Facebook, Twitter.

\section{The Galician newspapers on the online social networks: presence and positioning in the new communicative setting}

\begin{abstract}
The Galician newspapers prioritized the use of social networks as a distribution channel while they explore other applications in the new communicative scene. This study describes and classifies the activity and contents of the general newspapers of major diffusion in Galicia, together with the most veteran native digital, on three of the social networks with greater penetration in the Community (Facebook, Twitter and Youtube) according to their presence-promotion, the management of the conversation and the creation of contents of general interest. The results draw a media landscape still dominated by the traditional passive and one-way model in which the potential of social networks are limited to the showcase for promotion and the distribution of content.
\end{abstract}

Keywords: social networks, digital journalism, interactivity, Facebook, Twitter.

\section{Referencia normalizada}

LÓPEZ GARCÍA, Xosé y ALONSO RODRÍGUEZ, Sara (2013): "Los periódicos gallegos en las redes sociales virtuales: presencia y posicionamiento en el nuevo escenario comunicativo". Estudios sobre el Mensaje Periodístico. Vol. 19, Núm. 2 (julio-diciembre), págs.: 1001-1016. Madrid, Servicio de Publicaciones de la Universidad Complutense.

Sumario: 1. Introducción; 1.1. El impacto de las nuevas tecnologías: las redes sociales como motores de noticias; 1.2. Presencia y características de la Red en Galicia; 1.3. Redes sociales y cibermedios gallegos. 2. Objetivos y metodología. 3. Hipótesis. 4. Resultados; 4.1. Caracterización de la presencia-promoción; 4.2. Caracterización de la gestión-conversación; 4.3. Caracterización de la creación de contenidos de interés general. 5. Conclusiones. 6. Referencias. 


\section{Introducción}

\subsection{El impacto de las nuevas tecnologías: Las redes sociales como motores de no- ticias}

Ahora que sobrevivir parece ya el único negocio, Internet ha abierto las puertas a un nuevo modelo de información y relaciones sociales como espacio virtual que posibilita interacciones. En el marco de su proceso de convergencia y sus incidencias en múltiples esferas del quehacer periodístico (Salaverría, Avilés y Masip 2010: 58), los medios de comunicación se han visto obligados a aceptar una nueva economía atestada de competidores y de plataformas dónde la información tiene usos, significados y efectos muy distintos a los concebidos por el periodismo tradicional. En el nuevo ecosistema tejido por la arañera, la sociedad en red (Castells, 2002), todo el mundo es receptor y emisario, la experiencia de usuario, la vivencia y la interface de acceso son tan importantes como el propio contenido, y el mercado de oferta y demanda se ve destruido por la (sobre)abundancia digital, a pesar de que sus contenidos sean solo información de low cost. (Varela, 2011). En este contexto, las redes sociales se han erigido como herramientas que permiten modificar los procesos de creación, edición, distribución y consumo de contenidos de todo tipo con mayor facilidad, estando al alcance tanto de empresas y organizaciones como de usuarios particulares. Permiten el acceso a un mayor número de fuentes, así como el uso personalizado y una gestión independiente de los modelos de consumo colectivo, por lo que acompañan a un modelo de producción de contenido que alimenta al periodismo pero que ni lo sustituye ni lo anula. (Diezhandino, 2012: 60). Son, por tanto, la principal competencia de los medios pero también su mayor oportunidad dado el nuevo paradigma de participación ciudadana (Cardoso, 2010: 537).

En España, las redes sociales son ya uno de los ejes vertebradores del consumo digital y un fenómeno en continuo crecimiento. Según datos del informe del Observatorio Nacional de las Telecomunicaciones y de la SI en 2011, su penetración alcanza al 41,6\% de la población general (de 16 a 74 años) y al 55,6\% de los internautas. Su uso, más común entre personas con edades comprendidas entre los 16 y 24 años y una tasa de penetración del $89,4 \%$, se ha convertido en parte da la rutina diaria de la mayoría de la población. Datos de la Asociación para la Investigación de los Medios de Comunicación (AIMC) de febrero de $2012^{1}$, señalan que un $68,4 \%$ de los internautas declaran utilizar las redes sociales a diario, frente al $60,3 \%$ de hace un año, al $43,5 \%$ de hace dos y al $28,6 \%$ de hace tres. Los usuarios de redes sociales son internautas intensivos, pues navegan y utilizan más a fondo que el resto los servicios de Internet. Como principales actividades que realizan en las redes sociales destacan la introducción de contenidos y compartición con otros usuarios (55'7\%); ver, escuchar y leer contenidos sin descargarlos y sin comentarlos o etiquetarlos $(45,6 \%)$ y etiquetar o añadir comentarios a los contenidos $(32,5 \%)$. Especialmente significativo resulta que la lectura de noticias, periódicos y revistas sea la actividad ejercida por mayor por-

${ }^{1}$ http://download.aimc.es/aimc/f5g9/macro2011.pdf [Última fecha de consulta: 05/10/2012] 
centaje de usuarios de estas redes (84\%), seguida del consumo de fotografías $(82,1 \%)$ y la escucha de música $(80,1 \%)^{2}$.

Facebook lidera cada vez con más fuerza el espacio social digital con una combinación de intereses personales, profesionales y de contenidos. Desde su creación en 2004, creció de manera espectacular año tras año hasta superar el pasado 14 de septiembre los mil millones de usuarios activos ${ }^{3}$. En España continúa imbatible entre los internautas, aumentando su ventaja y consolidándose como uno de los grandes motores de tráfico de información. Con más de 17 millones de usuarios activos y una penetración del $89.8 \%$ entre los usuarios de Internet ${ }^{4}$, la red social de Mark Zuckerberg es dueña del flujo social. Al mismo tiempo y superados sus seis años de existencia, Twitter ya se ha convertido en una plataforma planetaria que ronda los 500 millones de usuarios. España es ya el segundo país europeo y el octavo del mundo con mayor porcentaje de usuarios activos ${ }^{5}$ gracias a que, en el último año, experimentó el mayor crecimiento de usuarios de todos los medios sociales, situándose como la segunda red social. Con una penetración del $36{ }^{\prime} 9 \%$ entre los internautas ${ }^{6}$, supera ya la barrera de los cinco millones de miembros, de los cuales, un $63 \%$ accede a diario 7 . Caracterizado por concentrar una corriente continua y en tiempo real de contenidos compartidos por los usuarios, funciona como un lugar dónde el nuevo algoritmo de la información es social y no tecnológico; un lugar donde la información es experiencia pública.

En ambas redes sociales, el perfil de los usuarios es mayoritariamente joven y masculino. Sin embargo, su comportamiento es substancialmente distinto respecto al flujo informativo. Según un estudio de Chitika ${ }^{8}$, el $28 \%$ de los usuarios Twitter están interesados en noticias mientras que en Facebook el porcentaje disminuye hasta el 18\%. En todo caso, las dos empiezan a ser una potente alternativa a buscadores, agregadores webs de recomendación social o suscripciones RSS. Además son los grandes aspirantes a convertirse en los líderes del marketing viral y de la gestión de la participación tanto de diarios como de televisiones. El análisis del tráfico de los medios informativos muestra que aún están lejos de la afluencia de visitantes de Google, pero se acercan cada vez más a Google News, Menéame y otros referentes.

2 Datos del Observatorio Nacional de las Telecomunicaciones y de la SI referentes a 2010.

3 http://tecnologia.elpais.com/tecnologia/2012/10/04/actualidad/1349353520_005170.html [Última fecha de consulta: 05/10/2012]

${ }^{4}$ Datos de la 14 ${ }^{\text {a }}$ Encuesta sobre Usuarios de Internet (AIMC) de febrero de 2012, en referencia al acceso en los últimos 30 días.

5 Datos que del informe de septiembre de 2012 de la empresa Globalwebindex. Disponible en http://globalwebindex.net/ [Última fecha de consulta: 05/10/2012]

6 Datos de la 14 a Encuesta sobre Usuarios de Internet (AIMC) de febrero de 2012, en referencia al acceso en los últimos 30 días.

7 Datos de la Cuarta Oleada (Abril 2012) del Observatorio de las Redes Sociales.

8 Chitika: "Twitterers Want Their News, and Want It Now", octubre 2009: http://insights.chitika.com/2009/twitterers-want-their-news/ [Última fecha de consulta: 05/10/2012] 


\subsection{Presencia y características de la Red en Galicia}

Según un estudio del Pew Research Center, los usuarios más educados y con mayor influencia y nivel de vida de cada país se concentran en las redes sociales, de forma que a la riqueza, vinculada a la disponibilidad de equipos y banda ancha móvil accesible, se une la actividad social y política de cada uno de ellos como características diferenciales. En Galicia, donde aún se sufre una enorme brecha digital respecto a los demás territorios del Estado, el análisis del perfil sociodemográfico de los usuarios en relación con el equipamiento y uso de las tecnologías de la información y de la comunicación en los hogares confirma que el género y el nivel de estudio son dos de las variables que modulan su comportamiento y pautas de uso.

Según datos de la $2^{\mathrm{a}}$ Ola del EGM (abril-mayo de 2012), un 51'2\% de la población gallega cuenta con conexión a Internet. A pesar de que esta cifra supone un incremento de más del doble con respecto a hace diez años, cuando la penetración de la red se situaba en torno al 19\%, Galicia continua siendo una de las comunidades con menor número de usuarios del Estado, situada solo por delante de Cantabria (46'8\%), Extremadura $(50,5 \%)$ y Asturias $(50,7 \%)$ y a mucha distancia de Madrid $(66,2 \%)$ y Cataluña $\left(66^{\prime} 7 \%\right)$. Atendiendo a los últimos datos ofrecidos por el INE', Galicia se también sitúa a la cola en cuanto a frecuencia a uso de la red, solo precedida por Extremadura: Un 58\% de la población gallega de entre 16 y 74 años accedió a Internet en los últimos tres meses y solo un 53,4\% en lo hace, al menos, una vez a la semana. Mientras, la brecha digital de género se materializa en un diferencial de 4' 2 puntos porcentuales que separa al 55,7\% de los hombres del 51,5\% de las mujeres gallegas que hacen uso de la $\operatorname{Red}^{10}$. En cuanto al uso de Internet según el nivel de estudios, se aprecia cómo la que a distancia digital entre España y Galicia se acentúa especialmente entre las personas que solo cuentan con estudios primarios (9,9 puntos) o únicamente con la primera etapa de educación secundaria (17,1 puntos). En el resto de estratos, la diferencia se sitúa por debajo de los 3,1 puntos $^{11}$.

Finalmente, es preciso mencionar al hábitat poblacional como otro de los factores clave en la configuración de la brecha digital gallega. Según la citada encuesta del INE, la diferencia entre hábitat urbano y rural en Galicia alcanza los 26 puntos porcentuales. Si bien en los núcleos urbanos se logra prácticamente la convergencia con el resto del territorio estatal, las diferencias se mantienen en el rural. El porcentaje de gallegos con conexión a la Red en los núcleos de población de más de 100.000 habitantes es del $60 ’ 2 \%$ y del $66,1 \%$ en las ciudades y villas de entre 50.000 a 100.000 habitantes, frente al $68 \% 1 \%$ estatal. En los núcleos de menos de 10.000 habitantes este indicador se sitúa en el 40 ' $1 \%$ en Galicia frente al 54'4\% de España.

9 Datos correspondientes a la Encuesta sobre Equipamiento y Uso de Tecnologías de la Información y Comunicación en los hogares 2011.

${ }^{10}$ Observatorio da Sociedade da Información e a Modernización de Galicia. Estado da Sociedade da Información de Galicia. Informe preliminar - marzo de 2011. Disponible en: http://www.osimga.org/export/sites/osimga/gl/documentos/d/20110302_informe_osimga_est ado_si.pdf [Última fecha de consulta: 05/10/2012]

${ }^{11}$ Ibid. 


\subsection{Redes sociales y cibermedios gallegos}

Prácticamente todos los medios de comunicación tradicionales en Galicia tienen representación en Internet. Desde que en octubre de 1.995 naciera el primer periódico digital gallego, el panorama mediático y tecnológico ha evolucionado significativamente. A pesar de que la incorporación de elementos propios de las nuevas plataformas virtuales avanza lentamente, el auge del uso de las redes sociales ha provocado que también los medios centren su interés en ellas. Si hace apenas dos años el reto para los cibermedios gallegos era implicarse en la blogosfera ofreciendo bitácoras desde su propio sitio web en los que crear comunidades de referencia para integrar al público en el propio medio, ahora el desafío consiste en saber posicionarse en las redes sociales en un contexto de especialización creciente.

La Voz de Galicia es hoy el periódico que realiza, a priori, la apuesta más importante por una presencia relevante en las redes sociales. Desde 2009, año del último estudio en profundidad de los cibermedios gallegos ${ }^{12}$, el rotativo coruñés creó, además de perfil en Youtube, un total de 27 cuentas en Twitter con las que segmenta su actividad en función de la edición local o de la sección temática. En Facebook, junto al perfil principal en el que se concentra toda la actividad de la Corporación Voz en esta plataforma, cuenta con otros 11 perfiles para cada una de las ediciones locales así como para Radio Voz, VTelevisión y suplementos especiales (como Voz Natura y Prensa Escuela). En el resto de medios, hay que destacar la incorporación de $\mathrm{El} \mathrm{Co}$ rreo Gallego y Faro de Vigo a Twitter y Facebook, donde hasta entonces únicamente tenía presencia La Voz de Galicia.

\section{Objetivos y metodología}

La investigación procura la definición de la naturaleza e idiosincrasia de las redes sociales en los cibermedios gallegos. A partir de este objetivo general, se pueden enumerar otros propósitos complementarios:

- Examinar las informaciones publicadas en las redes sociales de los distintos cibermedios a través de las características de cada elemento informativo, de los contenidos temáticos, de los recursos y elementos que las integran, de la respuesta que generan en los usuarios, etc. para describir las tendencias de la oferta informativa y su adecuación con las actuales formulaciones hipermedia e interactivas de la noticia en este tipo de plataformas.

- Identificar los elementos multimedia que acompañan a la información o que constituyen informaciones por si mismos para conocer el grado de multimedialidad de las distintas publicaciones en las redes sociales.

${ }^{12}$ El estudio "Cibermedios, blogs y redes sociales en Galicia 2009” fue realizado por el grupo Novos Medios de la Universidad de Santiago de Compostela. El presente proyecto, llevado adelante por Sara Alonso, se enmarcó en una Beca-Colaboración (Orden EDU/1868/2011 de 29 de junio de 2011), del Ministerio de Educación para el curso 2011-2012, y en los trabajos exploratorios para el proyecto de investigación "Innovación y desarrollo de los cibermedios en España-Arquitectura de la interactividad periodística en dispositivos múltiples: formatos de información, conversación y servicios". 
- Describir el grado de desarrollo de los cibermedios gallegos respecto a la puesta en funcionamiento de elementos interactivos y participativos y la respuesta por parte de los usuarios.

- Precisar si existe una estrategia definida en la gestión de las redes sociales.

Para la consecución de las metas propuestas, se optó por una perspectiva metodológica cuantitativa, mediante la aplicación del análisis de contenidos, para resolver las hipótesis que enmarcaban el fenómeno investigado. El enfoque elegido presenta, por tanto, un marcado carácter descriptivo, ya que permite analizar las características formales, periodísticas y multimedia de la presencia en las redes sociales de los cibermedios gallegos. Paralelamente, también presenta un matiz explicativo, al incidir en la busca de los porqués que desembocan en la práctica informativa que se desenvuelve en las redes sociales, al tiempo que se mueve en el ámbito correlacional, ya que facilita el establecimiento de relaciones entre los distintos medios analizados.

En este marco metodológico, se ha definido la población de análisis como aquella formada por los contenidos publicados en las redes sociales (Facebook, Twitter y Youtube) de tres periódicos gallegos con edición impresa y digital ( $L a$ Voz de Galicia, Faro de Vigo y El Correo Gallego) y del diario con versión electrónica exclusiva $G a-$ licia Confidencial. La selección de estas publicaciones se debe tanto a su liderato en cuanto a difusión diaria en Galicia ${ }^{13}$ como al uso más intenso que realizan de las redes sociales. Galicia Confidencial es además el nativo digital en gallego que mayor número de visitas registra ${ }^{14}$ (50.000 a la semana). La elección de las redes sociales responde a su mayor uso y penetración entre el público objetivo de estos medios. En algunos casos fue preciso elegir entre las diversas cuentas pertenecientes al medio, caso de La Voz de Galicia, escogiendo los perfiles que registran la actividad central del medio, tanto en Twitter como en Facebook.

Tras la selección, se optó por un muestreo por conglomerados, también llamado polietápico, en la procura de una mayor variedad temática y de la actualidad. Así, el análisis se realizó durante doce días comprendidos entre el 13 de febrero y el 13 de marzo de $2012^{15}$. Con la intención de que el estudio presentara una perspectiva integral se efectuó un volcado y observación de los contenidos publicados en tres turnos: a las 9:00, a las 15:00 y a las 00:00, recogiendo en cada análisis la información producida en el período que comprende cada franja horaria. Esa doble consulta permite que el trabajo aporte un grado de mayor concreción, al poder establecer comparaciones y fa-

${ }^{13}$ El Progreso quedó fuera del seguimiento, pues a pesar de ser el tercer periódico gallego con mayor difusión según el OJD (que non incluye en su medición a El Correo Gallego), su actividad en las redes sociales es casi inexistente.

${ }^{14}$ En el momento del inicio del análisis, el diario digital en gallego Praza Pública llevaba escasos diez días en la red. Su reciente lanzamiento y la consecuente falta de datos de audiencia impidió valorar su inclusión en el presente estudio.

${ }^{15}$ Los resultados corresponden a los días 13,14, 15, 23, 24, 25, 26, 27 y 28 de febrero y, 7, 8 9 de marzo de 2012. 
cilita el análisis de las actualizaciones efectuadas por los distintos medios durante el período de estudio.

Para establecer la base del registro y sistematizar la cuantificación, las unidades de análisis se dividieron en tres grandes bloques (presencia-promoción, gestión-conversación y creación de contenidos de interés) atendiendo tanto a aspectos comunes a las tres redes sociales como a características diferenciales de cada una de ellas con el propósito de conseguir unos resultados homogéneos, tanto cuantitativos como cualitativos. Finalmente, se empleó una estructura de recuento de cada variable en las correspondientes fichas de análisis.

\section{Hipótesis}

Partiendo de la observación directa y del marco teórico existente arredor del tema, se pueden establecer algunas hipótesis de partida:

- Los cibermedios gallegos no aprovechan las potencialidades y ventajas comunicativas de las redes sociales como escaparates y punto de encuentro para ampliar y fidelizar públicos.

- La mayor parte de los cibermedios no cuenta con una estrategia propia y definida para gestionar su actividad en las redes sociales, sino que su posicionamiento en esta plataforma se reduce a una simple translación de los contenidos presentes en su edición digital.

- Como consecuencia, la presencia de los cibermedios en las redes sociales virtuales se caracteriza por la irregularidad en la frecuencia de actualización, al tratamiento desigual de las temáticas y al uso indistinto de las diferentes plataformas, sin atender a sus especificidades, lo que, a la vez, impide el ejercicio de un periodismo más participativo y dialogante.

\section{Resultados}

La actividad de los cuatro cibermedios gallegos analizados durante el período señalado se traduce en un total de 590 post en Facebook, 2.002 tweets en Twitter y cinco vídeos en Youtube. Con una media de 166 tweets por día, Twitter es la red social más empleada. A la cabeza se sitúa Faro de Vigo, con un total de 670 tweets; seguido por La Voz de Galicia, con 588, El Correo Gallego, con 384 y Galicia Confidencial, con 360. Facebook aparece en segundo lugar con una media de 49 publicaciones diarias. La Voz de Galicia encabeza la divulgación de contenidos por esta vía, con un total de 253 post; seguida por Galicia Confidencial, con 182; Faro de Vigo, con 150 y El Correo Gallego, con tan solo cinco entradas. Así, La Voz de Galicia es el medio que, cuando menos cuantitativamente, desarrolla una actividad más intensa en las redes sociales.

El escaso volumen de información producida por los medios analizados en Youtube obligó a su descarte a la hora de realizar la interpretación de los datos recogidos, puesto no resultaba lo suficientemente representativa como para poder aventurar conclusiones. Sin embargo, es precisamente esta falta de contenidos la que permite constatar la escasa importancia que los cibermedios gallegos conceden, a día de hoy, al mayor portal de vídeos del mundo a la hora de posicionarse en las redes sociales virtuales. 


\subsection{Caracterización de la presencia-promoción \\ 4.1.1. Volumen de publicación por día y franja horaria}

En las redes sociales, la información y sus datos se dinamizan y conviven con un consumo en tiempo real y asincrónico que obliga a planificar y programar la publicación de la información para distintos consumos por el público.

En el caso de Facebook, el análisis de la totalidad de los post según la franja horaria en la que fueron difundidos muestra una concentración de la actividad entre las 9 y las 15 horas. En comparación con los datos de la AIMC respecto de las franjas horarias de mayor uso de Internet, vemos que, contrariamente, el mayor volumen de actividad de los internautas se concentra de 19 a 21 horas (47\%) y de 21 a 00:00 horas (56'1\%). En la actividad de los cibermedios en Facebook, los contenidos publicados entre las 00 y las 9 horas son prácticamente anecdóticos, pues en ninguno de los días analizados se superan los 5 post.

Si realizamos un análisis individual, La Voz de Galicia mantiene la pauta general de distribución temporal. Con una media de 21,03 post diarios, concentra el 59\% de su actividad en la franja de mañana, a cierta distancia de la franja de las 15:00 a las 00.00, que supone un 38’3\%. La franja de las 00:00 a las 9 de la mañana únicamente registra actividad en dos de los días analizados. La tendencia opuesta es la representada por Galicia Confidencial. Sus 15 post diarios de media presentan una distribución temporal menos homogénea que la del resto de medios y más acorde con la franja horaria de mayor uso de la Red. El cibermedio concentra mayoritariamente sus publicaciones en la franja de las 15:00 a las 00:00 en ocho de los 12 días analizados. En las restantes jornadas, los post se localizan básicamente en la franja de mañana.

En el caso de Twitter, el grueso de la actividad se produce entre las 9 de la mañana y las 15 de la tarde. Tan solo en dos de los días analizados (el 25 y 26 de febrero) el volumen de tweets publicados entre las 15 y las 00 horas supera a la actividad de la franja anterior. La cantidad de contenidos publicados en el horario nocturno es mínima, sin superar ninguno de los días los 20 tweets. Si comparamos estos resultados con los datos de la AIMC respecto de las franjas horarias de mayor uso de Internet, volvemos a comprobar que los medios no tienen en cuenta este comportamiento.

La Voz de Galicia vuelve a ser el medio con una estrategia de publicación más acorde con los horarios de mayor acceso a la Red aunque es Faro de Vigo el cibermedio que más intensamente difunde contenidos en Twitter, con una media de 56 tweets diarios. Su comportamiento sigue un patrón homogéneo en los doce días de análisis, con la práctica totalidad de los tweets publicados en la franja de mañana y a mucha distancia del resto de la jornada, lo que sugiere una intencionalidad por concentrar la actividad en determinados momentos. Galicia Confidencial se aparta del comportamiento del resto de medios y, de la misma forma que en el caso de Facebook, concentra su actividad en la franja de la tarde, cuando la Red registra un mayor uso por parte de los internautas. Sin embargo, es el medio que menor volumen de tweets publica con una media de 30 diarios.

\subsubsection{Volumen de contenido posteado y tuiteado con enlaces}

A la hora de determinar los distintos usos a los que se destina Facebook, es interesante comprobar la inclusión, o no, de enlaces a contenidos ajenos a su propio perfil en la 
red social. Los datos confirman que los cibermedios gallegos valoran la capacidad de establecer vínculos con otros sitios web. De los 590 post publicados por los cuatro cibermedios, 553 contenían, al menos, un hipervínculo (el 93\% del total de entradas) mientras tan solo 37 post fueron publicados sin ningún tipo de enlace.

Atendiendo al tipo de contenido se enlaza, se observa cómo los medios emplean Facebook como un redistribuidor de noticias con el que tratar de aumentar el tráfico de visitas a sus sitios web. Así, de los 553 post con vínculos, 525 tenían como destino la página web del medio (94\%) frente a los tan solo 28 que dirigían al usuario a portales o sitios web ajenos al periódico.

Twitter funciona como una herramienta de agregación de noticias. De ahí que el $91 \%$ de los tweets (1.823 de 2.002) contengan vínculos frente al 9\% restante (188) que solo incluye texto. En general, los cibermedios gallegos lo utilizan para promover contenido propio, producido y publicado en línea, pues el $97^{\prime} 45 \%$ de los tweets contienen enlaces que conducen a la web del cibermedio.

\subsubsection{Alcance territorial del contenido publicado}

La homogeneidad en la publicación de contenidos en las dos redes sociales y por parte de los cuatro medios queda patente al analizar el alcance territorial de las publicaciones. En todos los casos y con cifras semejantes, la información de proximidad es la gran dominadora.

En el caso de Facebook, de los 590 post analizados, 239 se referían a contenidos pertenecientes al ámbito gallego (40'5\%); 112 al estatal (19\%); 90 al local (15'25\%); 32 al internacional ( $\left.5^{\prime} 4 \%\right)$; 21 al europeo (3'56\%); nueve al comarcal (1'5\%) y tres al provincial (0'5\%). Así mismo, 84 de los post (14'2\%) no pudieron englobarse en ninguna categoría territorial al tratarse de contenidos de opinión, servicios, sugerencias a los seguidores, etc ${ }^{16}$.

En La Voz de Galicia, las informaciones de ámbito gallego y estatal representan más de la mitad del volumen total de post analizados. De los 253 publicados, 88 se refieren a informaciones del territorio gallego (34'78\%); 84 al estatal (33'2\%) 36 al internacional (14'22\%), 28 al local (11'06\%) y 14 al europeo (5'53\%). La proximidad es una característica aún más marcada en Faro de Vigo. Solo entre el contenido de ámbito gallego y local se suman casi 3/5 del total de post. Mientras, Galicia Confidencial presenta una clara vocación por los contenidos referidos a Galicia. De sus 182 post, $105\left(57^{\prime} 7 \%\right)$ pertenecen a esta categoría. El resto se reparten en las categorías correspondientes a la información gallega de proximidad: 20 post de información comarcal (10'98\%); 19 de local (10,43\%) y tres de provincial (1'65\%). La información relativa a acontecimientos de fuera de las fronteras gallegas tiene muy poca importancia. No existe ninguno de carácter internacional y tan solo 10 corresponden a contenidos del ámbito estatal ( $5{ }^{\prime} 5 \%$ ) y cinco al europeo (2'75\%). Finalmente, en el caso del El Correo Gallego, de los únicos cinco post publicados en Facebook, de los dos clasificables se refieren a informaciones de ámbito gallego.

${ }^{16}$ En la exposición de los resultados individualizados de cada periódico se obvian los post y tweets cuya inclusión en alguna de las diferentes categorías no fue posible. 
También en Twitter la información es básicamente gallega. De los 2002 tweets publicados, 713 hacían referencia a acontecimientos situados en territorio gallego (35'6\%); 391 aludían al ámbito local (19'53\%); 349 al estatal (17'43\%); 122 al internacional (6’09\%); 83 al europeo (4'15\%); 44 al comarcal (2'19\%) y ocho al provincial $\left(0{ }^{\prime} 4 \%\right)$.

En cuanto al comportamiento de cada medio, La Voz de Galicia se enmarca dentro de la tendencia general, aunque concede más importancia al contenido de carácter internacional que el resto de medios. De sus 588 tweets publicados, 171 pertenecen al ámbito gallego (29\%); 163 al estatal (27'72\%); 89 al local (15'13\%); 82 al internacional (13'94\%); 51 al europeo $\left(8^{\prime} 67 \%\right.$ ) y dos al comarcal. Faro de Vigo repite un esquema semejante. La mitad de sus 670 tweets pertenecen al ámbito gallego (181; o $27 \%$ ) y local (162; o 24’18\%). En la misma línea se sitúa la actividad de El Correo Gallego, aunque con una mayor concentración de los contenidos en las categorías pertenecientes a la información gallega. De sus 384 tweets, 146 se corresponden con informaciones de carácter gallego (38\%); 105 con contenidos del ámbito local (24’34\%); 91 del estatal (23'7\%); 10 del ámbito comarcal (2’61\%) y del provincial (0'5\%). Los contenidos extranjeros apenas suman un 7\% del total: 16 tweets pertenecen a la categoría de información del ámbito internacional y 10 del europeo. Galicia Confidencial es el medio que presenta unos contenidos más homogéneos desde el punto de vista de su alcance territorial, pues un total de 215 tweets, de los 360 publicados durante él período de análisis, se concentra en el ámbito gallego.

\subsubsection{Tipología del contenido publicado}

Igualmente, la tipología de contenido difundido por los periódicos gallegos en las redes sociales es coincidente. En Facebook, se observa una clara preponderancia del informativo, en correspondencia con el grueso de material propio de medios generalistas como los analizados. De los 588 post difundidos, 530 incluyen contenido informativo (90\%) y 40 contenido de opinión (6'80\%). La demanda de participación de los usuarios (encuestas, cuestionarios, concursos, opinión sobre determinado temas, etc) solo representa el 2'04\% del total (12 post) mientras que la información de servicios se limita a un anecdótico 1'36\% (8 post). El Correo Gallego es el único de los cuatro medios en que el contenido informativo no es el mayoritario. De sus cinco post, tres se refieren a contenidos de opinión y dos a contenidos informativos.

Twitter también se presenta como una plataforma para la difusión de contenido fundamentalmente informativo. Del total de 2.202 tweets publicados, 1.870 (el 93\%) se refieren a noticias de información general. El resto de contenidos son casi anecdóticos: 85 de ellos están relacionados con contenidos de opinión; 31 solicitan la participación de los usuarios para conocer su opinión sobre un tema o participar en una encuesta o concurso y 23 ofrecen información de servicios. Por medios, La Voz de Galicia difundió un total de 554 tweets informativos, 23 con contenidos de opinión, 10 con información de servicios y dos en los que demanda la participación de los usuarios. Mientras, 624 tweets difundidos por Faro de Vigo estaban vinculados con contenido informativo, 13 con información de servicios y ocho con contenidos de opinión. Aunque pocos respecto del contenido informativo, que representa el 93\% del 
contenido total, los 26 tweets que el diario vigués dedica a solicitar la participación y/o opinión de sus seguidores lo convierten en el medio que mayor preocupación muestra por establecer vínculos bidireccionales mediante este mecanismo. En el caso de $E l$ Correo Gallego destaca la total homogeneidad de sus publicaciones: Sus 384 tweets contienen únicamente material informativo. El análisis del timeline de Galicia Confidencial revela que de sus 360 tweets, 308 presentan contenido informativo (85'5\%), 51 contenido de opinión y un único tweet que solicita la participación dos seguidores.

\subsubsection{Twitter y la información de última hora}

La capacidad de permanente actualización y renovación convierte a Twitter en una plataforma perfecta para la difusión de información de última hora, funcionando como un sistema de alerta temprana. Sin embargo, del total de 2.002 tweets producidos durante el período de análisis, tan solo 27 pueden encuadrarse en esta categoría. El medio que realiza un mayor aprovechamiento de esta funcionalidad es Faro de Vigo, especialmente para la retransmisión en directo de acontecimientos deportivos. De hecho, la mayor parte de los tweets de última hora de este medio ( 8 de los 17 de su autoría) corresponde al comentario de un partido de balonmano disputado por el equipo vigués Academia Octavio contra el Cuatro Rayas Valladolid el 7 de marzo de 2012. En el caso de Galicia Confidencial los tweets clasificables en este grupo, siete en total, suelen ir precedidos de términos como "URXENTE" y funcionan como un aviso de los contenidos que, en un período corto de tiempo, el usuario podrá encontrar en la página web del medio. Por ejemplo, el del día 27 de febrero: "URXENTE: A Executiva do BNG decide expulsar aos concelleiros desta formacion en Cee. En breve en http://bit.ly/6rmah7'). Cuando se trata de una información de última hora de la que non se disponen de más datos, Galicia Confidencial lo señala con el indicativo "AVANCE". Por ejemplo, en el caso del día 9 de marzo: "AVANCE \#FernandoBlanco nega implicación na\#OpCampéon: «Nunca presionei para que cobrara subvención' e Dorribo 'responderá nos tribunais»"). La Voz de Galicia únicamente publica tres tweets con estas características mientras que en El Correo Gallego no tuitea ningún contenido o información que pueda englobarse dentro de esta categoría. En los tres medios que si lo hacen, es habitual que estos tweets no contengan vínculos, ni a contenido propio ni ajeno, por tratarse de datos que se acaban de conocer.

\subsubsection{Uso del hashtag}

El símbolo hashtag (\#) es empleado antes de palabras claves para clasificar tweets y facilitar buscas en Twitter. Señala que un tweet, y por extensión la historia enlazada, está relacionada con un tema o noticia en curso, lo que permite que, empleados con criterio (eventos, procesos electorales, campañas, accidentes, catástrofes, etc.) ayuden a monitorizar la conversación global y a encontrar fuentes relevantes. Así mismo, pueden aumentar la posibilidad de que personas que no estuvieran siguiendo la cuenta del medio, accedieran a ella a través del hashtag y acabaran leyendo la noticia en su página web.

Su uso es desigual según el medio analizado pero, en general, se trata de una funcionalidad desaprovechada. De los 2.002 tweets publicados en total por los cuatro pe- 
riódicos analizados, 527 incluían, al menos, un hashtag, lo que tan solo representa el $26 ’ 32 \%$ del total de tweets contabilizados. Por medios, Faro de Vigo es el que realiza un empleo más intenso del etiquetaje de contenido, con un $37 \%$ de sus tweets que contienen, al menos, un hashtag, como \#Xatcobeo (13 de febrero), en relación con el lanzamiento del primer satélite artificial gallego o \#Rosalia175 (24 de febrero), con motivo de la celebración del 175 aniversario del nacimiento de Rosalía de Castro. En el caso de Galicia Confidencial el aprovechamiento de esta herramienta se traduce en el etiquetado de una cuarta parte del total de volumen publicado. La Voz de Galicia apenas emplea el etiquetaje de contenio pues tan solo 41 de sus 588 tweets contienen, como mínimo, un hashtag. El Correo Gallego no lo emplea ni una sola vez en ninguno de sus 384 tweets.

\subsection{Caracterización de la gestión-conversación \\ 4.2.1. Evolución del número de fans y seguidores}

En Facebook, La Voz de Galicia registra el mayor número de fans, con una evolución de captación de nuevos usuarios ascendente y continua, pasando de los 26.353 del 13 de febrero a los 26.999 del 9 de marzo ${ }^{17}$. En el caso de Faro de Vigo, el segundo periódico con mayor número de seguidores, también se observa un crecimiento, aunque mucho más lento y pequeño. El periódico olívico experimentó un aumento de 142 seguidores, alcanzando los 6.524 el último día analizado. El Correo Gallego posee el menor número de seguidores en correspondencia con su casi nula actividad en esta rede social. Tanto su cuenta, como la de Galicia Confidencial incorporaron 62 nuevos usuarios, alcanzando los 3.838 y los 6.001 seguidores respectivamente.

También en Twitter es La Voz de Galicia quién concentra un mayor volumen de seguidores, registrando un crecimiento aún más notable y fuerte que en Facebook. Su aumento se cuantifica en 3.062 seguidores, alcanzando los 32.245 el último día de análisis. En el resto de medios, el crecimiento de seguidores, por orden de importancia se traduce en un aumento de 716 en Faro de Vigo, 316 en El Correo Gallego y 238 en Galicia Confidencial, que con 2.895 cuenta con el menor número de seguidores. Si comparamos la cifra de seguidores con el número de cuentas a las que siguen los medios observamos una total descompensación. Especialmente grave es el caso de $E l$ Correo Gallego cuyo comportamiento refleja que no realiza una discriminación entre sus seguidores, sino que simplemente opta por no seguir a ninguno. Durante el periodo analizado tan solo comenzó a seguir a una nueva cuenta, pasando de cinco a seis, todas ellas vinculadas, además al propio medio, pues se corresponden con diferentes secciones del periódico (Tendencias, Comarcas, Deportes, Sección Local de Santiago...) y al Galicia Hoxe, periódico perteneciente al mismo grupo editorial que El Correo Gallego (Editorial Compostela).

\subsubsection{Respuestas al feed-back}

Un aspecto clave en el análisis de la actividad de los cibermedios en las redes sociales es la interacción y los vínculos que establecen con sus seguidores. En el caso de

${ }^{17}$ La comparativa se realizó a partir de los seguidores registrados diariamente en la observación correspondiente a la franja de tarde (a las 15.00 horas). 
Facebook, es posible medir este comportamiento mediante la observación del número de "Me gusta" que el medio otorga a los comentarios realizados por sus seguidores en su tablón y a través de la cantidad de respuestas que da a las opiniones, sugerencias o demandas vertidas por los internautas. Atendiendo a estos parámetros, comprobamos como tan solo se registra un "Me gusta" y seis respuestas a los comentarios de los seguidores entre los cuatro medios, lo que revela la escasa conciencia de los diarios acerca de la importancia de crear y mantener un feed-back con sus seguidores.

Entre las contestaciones de los cibermedios, dos tienen la autoría de $\mathrm{La} \mathrm{Voz} \mathrm{de} \mathrm{Ga-}$ licia y una de ellas atiende a una petición de corrección, lo que indica, a lo menos, una mínima preocupación por satisfacer las reclamaciones del público lector: "Hemos corregido el titular aquí en Facebook, tal y como habéis sugerido unos cuantos con toda la razón. Buenas noches a todos" (13/02/2012). Faro de Vigo es responsable de un "Me gusta" y cuatro respuestas. Uno de estos comentarios complementa a una información y, a la vez, actúa como sugerencia para los usuarios que previamente realizaran comentarios en ese post: "En Faro de Vigo os informamos de que hemos creado en Twitter el hashtag \#ungallegoenelespacio. Si queréis uniros a los cientos de felicitaciones que la Universidad de Vigo está recibiendo en esta red social, usa la etiqueta" (13/02/2012). El Correo Gallego y Galicia Confidencial non establecen ningún tipo de interacción con sus seguidores en Facebook.

En el caso de Twitter, podemos señalar tres mecanismos para medir la importancia que el medio otorga a la interacción: la contestación de tweets procedentes de otros usuarios, la marcación de tweets favoritos de otros y el retuiteo de la actividad de otras cuentas. Éste último es la práctica más significativa y habitual, pues funciona como una herramienta de recomendación que permite a los cibermedios reenviar un tweet de otro usuario a su audiencia de noticias, introduciendo nuevas voces e ideas, o ampliar la información sobre un tema a través de la inclusión de otras fuentes. Sin embargo, la interacción es muy limitada. De los 2.002 tweets, tan solo 144 son retuiteos, 99 tweets de sus seguidores fueron marcados como "Favoritos" y 33 se corresponden con respuestas a alguna petición u opinión de algún usuario.

Por medios es Faro de Vigo el que otorga una mayor atención a sus seguidores. El $14 \%$ de sus tweets son retuiteos de otros usuarios (94 de los 670 totales); 99 tweets de sus seguidores aparecen marcados como "Favoritos", la mayor parte de ellos surgidos como respuesta a alguna solicitud de participación enviada por el propio periódico, y 16 tweets fueron difundidos en contestación a demandas de distintos usuarios. Como paradigma de la despreocupación por interactuar con el público seguidor aparece $E l$ Correo Gallego: No envía ningún retweet, no marca ningún "Favorito" ni envía ninguna respuesta en todo el período analizado.

\subsection{Caracterización de la creación de contenidos de interés general 4.3.1. Influencia del contenido en el comportamiento de los internautas}

Las redes sociales mantienen y aumentan el poder de marca y el valor de los medios informativos, a diferencia, por ejemplo de los buscadores, gracias a su sistema de recomendaciones personales, con el que aumentan la economía de la afectividad. Uno de los mejores indicativos de la optimización social es la respuesta del público a tra- 
vés de sus comportamientos respecto de los contenidos de los medios. Prácticas como los "Me gusta"; los comentarios o la compartición de post de otros usuarios son herramientas que permiten a los medios "escuchar" a sus seguidores, integrarlos en el proceso informativo y gestionar su identidad y participación.

En el caso de los seguidores en Facebook, se observa un alto grado de participación, superando con creces la actividad de los periódicos: Los 590 post de los medios generaron un total de 6.359 "Me gusta", 2.173 post compartidos y 1.889 comentarios. Con diferencia, los seguidores de La Voz de Galicia son los más activos, en correspondencia con el volumen de contenidos publicado por el diario corunés. En total, los fans de La Voz generaron 4.801 "Me gusta"; realizaron 1.736 comentarios y compartieron un total de 2.183 veces los 253 post del medio.

En lo referente a la observación de la influencia que el contenido difundido por los medios gallegos tiene sobre sus seguidores y, por extensión, en la masa de usuarios de Twitter, el retuiteo resulta también muy útil. En general, se trata de usuarios muy activos, aparentemente interesados por las informaciones ofrecidas por los periódicos. De forma absoluta, su actividad dobla a la de los medios: frente a los 2.002 tweets publicados por los cuatro cibermedios se generaron un total de 4.040 retweets, es dicir, prácticamente el doble. Es importante observar como los picos de mayores retuiteos no coinciden necesariamente con un aumento de tweets por parte de los medios, lo que permite suponer que no existe una relación simplemente proporcional entre unos y otros. En el caso de La Voz de Galicia sus 588 tweets generaron un total de 3.013 retweets, lo que significa que el reenvío de los usuarios de este contenido multiplica por cinco el volumen inicial de tweets del periódico. Esto es, cada tweet de La Voz de Galicia genera una media de cinco retweets. No sucede lo mismo en el caso de Faro de Vigo. El volumen de tweets producidos por el medio (670) es mayor que el de los retweets de sus usuarios (564). Tendencia que se repite también en la actividad de $\mathrm{El} \mathrm{Co}$ rreo Gallego, cuyo volumen de tweets (384) dobla al de retweets (154) y es superior a éstos en los 12 días analizados. Finalmente, Galicia Confidencial logra casi el equilibrio entre o contenido propio generado y su difusión entre los seguidores, pues sus 360 tweets obtuvieron 339 retweets.

\section{Conclusiones}

El análisis presentado demuestra que el posicionamiento de los periódicos gallegos ante las redes sociales es muy dispar. No obstante, es posible establecer una serie de características comunes:

- Las redes sociales se conciben como canales a través de los que distribuir información más que como un punto de encuentro con el que atraer y fidelizar a usuarios que, de otra manera, quizás no conocerían su oferta informativa. La tipología de actividad llevada a cabo en Twitter es un reflejo de su actividad diferencial. Funciona como un redistribuidor de información, como un canal RSS para los consumidores de noticias, con enlaces que dirigen el tráfico hacia las páginas web de los medios, y no tanto como una conversación colectiva o una red social propiamente dicha. Lo mismo sucede en Facebook, dónde la práctica totalidad de los contenidos incluyen hipervínculos que conducen a los lectores hacia los sitios web de los medios. 
- Los contenidos difundidos en las redes sociales se encuadran básicamente dentro del territorio gallego. Las informaciones de carácter local, comarcal y nacional copan la mayor parte del material publicado por los cibermedios gallegos frente a los estatales e internacionales.

- En todos los periódicos y redes sociales predominan los contenidos informativos, en detrimento de los géneros de opinión, la información de servicios o los contenidos que reclamen la participación de los lectores.

- El número de usuarios que siguen los perfiles de los cibermedios gallegos experimenta un crecimiento continuo aunque que desigual en función del medio, tanto en Facebook como en Twitter. El alto grado de participación del público mediante el comentario y reenvío de la información publicada por los diarios demuestra el interés creciente de los usuarios por su actividad en las redes sociales.

- No existe interés por abrir conversación con los usuarios y lectores. Los cibermedios gallegos desaprovechan las potencialidades relacionadas con la creación de vínculos con el público receptor al prestar una escasa atención a la utilización de los elementos de remisión y redifusión. Cuando los periodistas y el propio medio participan en la conversación y se convierten en elementos activos de las redes sociales y comunidades que crean, se mejoran muchos aspectos del proceso informativo: El debate, pero también la relación periodística con fuentes y público, la incorporación de criterios de la comunidad, la fidelización, la personalización y la transparencia.

- En el caso de Twitter, se desperdicia su capacidad de permanente actualización para adelantar datos e informaciones de última hora que conduzcan a los usuarios, posteriormente, hacia las páginas webs de los medios. La práctica totalidad de los contenidos enlazados son noticias producidas y colgadas en línea previamente.

- Tampoco se saca partido de especificidades propias de las redes sociales, como la posibilidad de mejorar la visibilización de los contenidos a través de su etiquetaje en Twitter.

En definitiva, los periódicos gallegos todavía están explorando posibilidades y únicamente priorizan las redes sociales su faceta como canal de distribución. La gestión de la conversación que generan sus mensajes, así como las otras potencialidades de las redes, aún no figuran entre las prioridades de los principales cibermedios de Galicia.

\section{Referencias}

ASOCIACIÓN PARA LA INVESTIGACIÓN DE MEDIOS DE COMUNICACIÓN (AIMC) (2012): "14 a Encuesta a Usuarios de Internet (Navegantes en la Red)", febrero 2012. Disponible en: http://download.aimc.es/aimc/f5g9/macro2011.pdf. [Última fecha de consulta: 05/10/ 2012]

CARDOSO, Gustavo (2010): Los medios de comunicación en la sociedad en red. Filtros, escaparates y noticias. Barcelona, UOC Ediciones.

CASTELLS, Manuel (2002): A Sociedade em Rede. A Era da Informaçao. Economia, Sociedade e Cultura., Volume I. Lisboa, Fundaçao Calouste Gulbenkian. 
DIEZHANDINO, Pilar (2012): "El valor añadido del periodismo que viene", en DIEZHANDINO, Pilar: El periodista en la encrucijada. Barcelona, Ariel, pp. 4575.

ESTUDIO GENERAL DE MEDIOS (EGM) (2012): “Audiencia de Internet en el EGM Abril-Mayo 2012", en http://www.aimc.es/-Audiencia-de-Internet-en-elEGM-.html [Última fecha de consulta: 11/10/2012]

INSTITUTO NACIONAL DE ESTADÍSTICA (INE) (2011): "Encuesta sobre equipamiento y uso de tecnologías de la información y comunicación en los hogares" (2011). Disponible en: http://www.ine.es/jaxi/menu.do?type=pcaxis\&path=/t25 /p450/a2011/\&file=pcaxis [Última fecha de consulta: 05/10/2012]

OBSERVATORIO DE LA REDES SOCIALES, Cuarta Oleada Abril (2012): Disponible en http://www.slideshare.net/TCAnalysis/4-oleada-observatorio-de-redes-sociales [Última fecha de consulta: 05/10/2012]

OBSERVATORIO NACIONAL DE LAS TELECOMUNICACIONES Y DE LA SI. Novembro 2011 "Informe anual de los contenidos digitales en España 2011. Industria y hábitos de consumo". Disponible en: http:/www.ontsi.red.es/ontsi /sites/default/files/informe_contenidos_digitales_2011_v7.pdf [Última fecha de consulta: 05/10/2012]

PEW RESEARCH CENTER (2011): "Global Digital Communication: Texting, Social Networking Popular Worldwide", diciembre 2011. Disponible en: http://www.pewglobal.org/2011/12/20/global-digital-communication-texting-social-networkingpopular-worldwide/1/ [Última fecha de consulta: 05/10/ 2012]

SALAVERRÍA, Ramón, GARCÍA AVILÉS, José Alberto, y MASIP, Pere (2010): "Concepto de convergencia periodística". En LÓPEZ, Xosé y PEREIRA, Xosé (Coords.): Convergencia Digital. Reconfiguración de los Medios de Comunicación en España. Santiago de Compostela, Universidad de Santiago de Compostela, pp. 41-64.

VARELA, Juan (2011): "Redacciones abiertas con participación de calidad", en Periodistas 21 . http://periodistas 21. blogspot.com.es/2011/04/redacciones-abiertascon-participacion.html [Última fecha de consulta: 05/10/2012] 